\title{
Clinical and Pathological Study of Polyomavirus-Associated Nephropathy after Renal Transplantation
}

\author{
JI Shu-ming ${ }^{1 *}$, Xie Ke-nan ${ }^{1}$, Chen Jin-song ${ }^{1}$, Wen Ji-qiu ${ }^{1}$, Cheng Dong-rui ${ }^{1}$, LI xu ${ }^{1}$, NI Xue-feng ${ }^{1}$ and Liu Zhi-hong $^{2}$ \\ ${ }^{1}$ National Clinical Research Center of Kidney Diseases, Jinling Hospital, China \\ ${ }^{2}$ Nanjing University School of Medicine, China
}

"Corresponding author: Shu-Ming JI, National Clinical Research Center of Kidney Diseases, Jinling Hospital, 305 East Zhong Shan Road, Nanjing 210002, China, Tel: 86-25-80860118; E-mail: jishuming2009@163.com

Rec date: Apr 19, 2014, Acc date: May 20, 2014, Pub date: May 22, 2014

Copyright: (c) 2014 Shu-Ming $\mathrm{Jl}$, et al. This is an open-access article distributed under the terms of the Creative Commons Attribution License, which permits unrestricted use, distribution, and reproduction in any medium, provided the original author and source are credited.

\begin{abstract}
Polyomavirus infection has emerged as an important cause of polyomavirus-associated nephropathy (PVAN) leading to allograft dysfunction and loss. The aim of this study is to investigate pathological features and clinical characteristics of PVAN. We prospectively investigated 351 renal allografts performed in Jinling Hospital. PVAN was diagnosed by light microscopic examination and a positive immunohistochemistry staining of anti-SV40 large T antibody in a biopsy specimen. 31 patients were diagnosed with PVAN (8.8\%). The patients with PVAN typically presented as allograft dysfunction with an asymptomatic rise in serum creatinine about 3 to 39 months posttransplant. Urinary decoy cells were positive in 4 patients (12.9\%). The histologic changes of PVAN are not pathognomonic and can be mistaken for allograft rejection, i.e. tubulointerstitial nephritis with varying degrees of inflammatory infiltrates, tubulitis, tubular atrophy and fibrosis. Typical findings on histology are focal interstitial mononuclear inflammatory cell infiltrates, presence of plasma cells, necrotic tubular epithelium, and presence of homogenous intranuclear inclusion bodies. Immunohistochemistry with SV40 staining is positive in allograft. CD3, CD4, CD8, CD68 positive cells are increased in PVAN group than the non-PVAN group, but none of HLA-DR and IL-2R expression is positive in PVAN patients. MPA AUC 0-12 and TAC trough levels are increased in PVAN group than non-PVAN group at biopsy. We have treated 31 patients with biopsy-proven PVAN with leflunomide. Increased intensity of immunosuppression appears to increase the likelihood of PVAN. The definitive diagnosis of PVAN requires renal biopsy. Immunohistochemistry with SV40 staining has been used as an indirect method to document the presence of PVAN.
\end{abstract}

Keywords: Renal transplantation; Biopsy; Polyomavirus; Polyomavirus-associated nephropathy; Pathology

\section{Introduction}

Polyomavirus-associated nephropathy (PVAN) is one of the most serious complications after renal transplantation $[1,2]$. PVAN has not attracted the attention of clinical transplant physicians over the years. The issue has become more important in recent years, as several new immunosuppressant agents have been introduced for treating renal transplant recipients. PVAN is an aggressively destructive disease occurring in up to $8 \%$ of kidney allograft recipients [3], with one-year graft-loss rate ranging from $30 \%$ to $65 \%$ [4]. PVAN has become an important cause of allograft loss in renal transplantation, but only limited information is available regarding its pathogenesis. Uncertainty about diagnostic procedures and paucity of effective interventions lead to the high rate of graft loss among renal transplant recipients with PVAN. Currently lack of effective therapeutic drugs, high rates of failure and loss caused by PVAN, and the leading risk factor for PVAN and renal allograft loss are still difficult problems in dire need of resolve [5-7]. PVAN diagnosis mainly depends on the renal allograft biopsy. Polyomavirus infection can cause acute tubular necrosis, but early diagnosis of PVAN and use of effective treatment can prevent irreversible allograft damage caused by virus and improve prognosis of PVAN. Therefore early diagnosis of PVAN has become current research focus [8-10]. Celik et al. took the initiative in regular biopsy after renal transplantation, which contributed to timely diagnosis of
PVAN [11]. Currently transplant centers have begun to carry out some researches on polyomavirus infection after renal transplantation, but clinical and pathological characteristics studies on PVAN have rarely been reported. Renal allograft dysfunction and even allograft loss caused by PVAN are lack of enough attention. The aim of this study is to investigate pathological features and clinical characteristics of PVAN.

\section{Materials and Methods}

\section{Patients}

Among 351 cases of patients diagnosed with decreased graft functions by renal allograft biopsy in our unit, from August 2005 to January 2013 period, 31 patients met the inclusion criteria.

\section{Inclusion criteria}

I. More than 3 months after renal transplantation, increased serum creatinine ( $\mathrm{SCr} \geq 1.5 \mathrm{mg} / \mathrm{dl}$ or greater than $30 \%$ in increase of serum creatinine values above the baseline), and slow clinical progression (increase in serum creatinine $<1 \mathrm{mg} /$ week), accompanied with or without hematuria or proteinuria; II. Vascular and post-renal factors in renal allograft were excluded by color doppler ultrasound. All patients were carried out with blood routine, urine routine, liver and renal function, blood coagulation function and concentration measurements of cyclosporine A (CsA ) or FK506 (tacrolimus, Tac) and mycophenolate mofetil (MMF) in blood before biopsy. Decoy cells 
Citation: Shu-Ming JI, Ke-nan X, Jin-song C, Ji-qiu W, Dong-rui C, et al. (2014) Clinical and Pathological Study of Polyomavirus-Associated Nephropathy after Renal Transplantation. J Transplant Technol Res 4: 132. doi:10.4172/2161-0991.1000132

Page 2 of 6

were detected in urine. All patients gave their written informed consent.

\section{Pathological study}

Renal graft biopsy was carried out under the guidance of $\mathrm{B}$ ultrasound. The specimens were qualified if the renal tissues contained 2 vessels and more than 10 renal glomeruluses, and were confirmed with conventional light microscopy and immunofluorescence examination. Light-microscopic staining included HE, PAS, PASM and Masson trichrome staining. The pathological diagnosis of allograft was in accordance with Banff 97 criteria [12].

\section{Immunofluorescence}

Direct fluoreslent assay was used to observe the deposition intensities and sites of IgG, IgA, IgM, C3, C4, C1q, and C4d. All frozen tissue sections were confirmed with $\mathrm{C} 4 \mathrm{~d}$ immunofluorescence staining, and the staining intensities were in accordance with Banff 2001 criteria, in which C4d negative was defined as $<25 \%$ of PTC C4d deposition, reversible focal positive was defined as $25 \%$ to $50 \%$ of PTC C4d deposition and C4d asystematic positive was defined as $>50 \%$ of PTC C4d deposition [13].

\section{Immunohistochemistry}

Enrolled patients were carried out with CD4, CD8, CD68, IL-2R, HLA-DR and POLYOMAVIRUS virus immunohistochemical staining. Paraffin immunohistochemistry and Envision two-step method were applied: the paraffin sections were deparaffinized by normal method, treated by $3 \%$ hydrogen peroxide to inhibit the endogenous peroxidase, placed into a pressure cooker with $1 \mathrm{ml}$ EDTA solution for antigen retrieval, blocked in normal $10 \%$ bull serum solution, washed and added with monoclonal mouse anti-human CD3 (1:100), CD68 (1:100), CD20 antibody (1:100) and HLA-DR antibody (1:50), respectively, incubated at room temperature for 2 hours, added with Envision and incubated at room temperature for 40 minutes, colored with $\mathrm{DAB}$ for 5 minutes, counterstained with hematoxylin for 2 minutes, dried and sealed with neutral gum. Read under a Nikon 8100 microscope. SV240 large T antigen was used for positioning (antipolyomavirus antibody staining).

The infiltration degrees of CD4, CD8, IL-2R, and CD68-positive cells were classified according to the following criteria: 16 high power fields with a total area of $1 \mathrm{~mm}^{2}$ were randomly selected for counting, The sum of counting greater than 300 was defined as 3 points, 200 to 300 was defined as 2 points, 100 to 200 was defined as 1 point, and less than 100 was defined as 0 point.

HLA-DR expression was judged on the basis of 50 tubular crosssections, and the percentage of HLA-DR positive tubular in the whole tubular. More than $50 \%$ was defined as 3 points, $25 \%$ to $50 \%$ was defined as 2 points, $10 \%$ to $25 \%$ was defined as 1 point, and less than $10 \%$ was defined as 0 point.

\section{Diagnosed criteria of PVAN}

SV240 large T antigen was located in the nuclear of renal tubular epithelial cell. Positive expression was defined as brown color and negative cells were not detected. PVAN was diagnosed through their positive expression in combination with HE staining $[2,14,15]$.

\section{Results}

Clinical data: Renal allograft biopsy was implemented in 351 patients followed by polyomavirus large $\mathrm{T}$ antigen staining. 31 positive cases were found and diagnosed as PVAN with incidence rate of $8.8 \%$. According to staining results of polyomavirus large $\mathrm{T}$ antigen staining of renal allograft biopsy, the patients were divided into two groups: PVAN group $(n=31)$ and non-PVAN group $(n=320)$. There was no significant difference in gender, age, and serum creatinine levels when performing renal biopsy (Table). However, the incidences of acute rejection, FK506 nephrotoxicity, pulmonary infection, and chronic renal allograft dysfunction before biopsy in PVAN group were obviously higher than that in non-PVAN group. All the 31 patients had slowly progressive increases in serum creatinine at the onset of disease, without hematuria, proteinuria, and other specific clinical symptoms.

\begin{tabular}{|l|l|l|l|}
\hline & PVAN group & Non-PVAN group & P \\
\hline Gender (Male/Female) & $20 / 11$ & $208 / 112$ & $>0.05$ \\
\hline Age (yr.) & $36.8 \pm 10.1$ & $37.4 \pm 11.2$ & $>0.05$ \\
\hline Postoperative onset time (mon) & $9.25 \pm 3.7$ & $11.4 \pm 5.6$ & $>0.05$ \\
\hline Historical events & & & $22(6.9 \%)$ \\
\hline Acute rejection (n/\%) & $2(6.5 \%)$ & $16(5.0 \%)$ & $>0.05$ \\
\hline FK506 nephrotoxicity (n/\%) & $4(12.9 \%)$ & $21(6.6 \%)$ & $<0.05$ \\
\hline Pneumonia (n/\%) & $6(19.4 \%)$ & & $<0.05$ \\
\hline Immunosuppressive schema & & $60(18.8 \%)$ & \\
\hline CsA+MMF+Pred (CMP) & $6(19.4 \%)$ & $188(58.8 \%)$ & $>0.05$ \\
\hline Tac + MMF+Pred (TMP) & $20(64.5 \%)$ & $72(22.5 \%)$ \\
\hline Tac + MMF+Pred+TII (TMPT) & $5(16.1 \%)$ & $1.1 \pm 1.2$ & $>0.05$ \\
\hline Serum creatinine level (mg/dl) & $2.7 \pm 1.5$ & & $>0.05$ \\
\hline $\begin{array}{l}\text { BKVAN: BK virus associated nephropathy } \\
\text { CsA: cyclosporine A, MMF: mycophenolate mofetil,Tac: tacrolimus,Pred: prednisone }\end{array}$ & $<0.05$ \\
\hline
\end{tabular}


Citation: Shu-Ming JI, Ke-nan X, Jin-song C, Ji-qiu W, Dong-rui C, et al. (2014) Clinical and Pathological Study of Polyomavirus-Associated Nephropathy after Renal Transplantation. J Transplant Technol Res 4: 132. doi:10.4172/2161-0991.1000132

Page 3 of 6

TII: Tripterygium Wilfordii

Table: Comparison of baseline data between two groups.

Decoy cells were found in urine of 4 patients (positive rate of $12.9 \%)$, and decoy cells in urine were detected for 3 times. The results showed that there were a large number of visible exfoliated cells and scattered polyomavirus-positive cells. Green fluorescence could be visible in the cell nuclear. The results are as follows: urinary protein $<0.10 \mathrm{~g} / 24$ hour, urinary sediment $\mathrm{RBC}<10000.0 / \mathrm{ml}$, white blood cells $<0-1 / \mathrm{HP}$; NAG enzyme $>42.1 \mathrm{U} / \mathrm{g}$. $\mathrm{Cr}(<17.0 \mathrm{U} / \mathrm{g}$. Cr $), \mathrm{RBP}>$ $11.94 \mathrm{mg} / \mathrm{L}, \quad \mathrm{KIM}-\mathrm{l}>11.46 \mu \mathrm{g} / \mathrm{L}(<0.4 \mu \mathrm{g} / \mathrm{L}), \quad \mathrm{IL}-1822.93 \mathrm{ng} / \mathrm{L} \quad(<20$ $\mathrm{ng} / \mathrm{L})$, and NGAL $>25.18 \mu \mathrm{g} / \mathrm{L}(<20 \mu \mathrm{g} / \mathrm{L})$.

Pathological features of PVAN: The typical pathological features of PVAN were as follows: different phenotypes of basophilic polyomavirus virus inclusion bodies appeared in renal tubular epithelial cells, infected renal tubular epithelial cells exfoliated towards renal tubular lumen, resulting in bare spot on the base membrane (Figure 1A, 1B). Interstitial was prominently presented in multifocal gathered infiltrating cells (mainly mononuclear cells and plasma cells), and their surrounding tubulars often had tubulitis, multifocal thickening and atrophy of tubular basement membrane (Figure 1C, 1D). Later pathological changes mainly included fibrosis, and a few of polyomavirus -infected cells (Figure 1D).

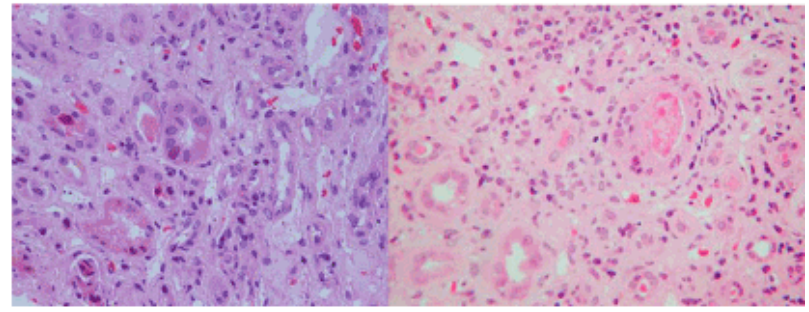

A: Multiple viral inclusions could $\mathbf{B}$ : Multifocal tubular atrophy and be seen in the renal tubular basement membrane thickening be seen in the renal tubular basement membrane thickening, epithelial cells, and the tubular focal tubular epithelial
epithelial cells were took off to
growth in flat $(\times 200)$ epithelial cells were
the lumen $(\times 200)$

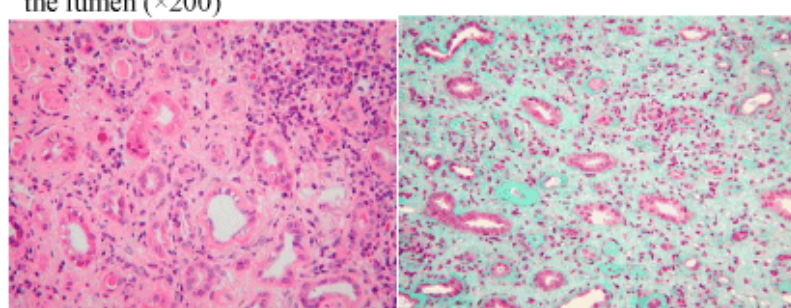

C: More mononuclear cells and plasma cells were infiltrated, small focal neutrophil distribution and a small amount of peritubular capillary inflammation could be seen $(\times 400)$

Figure 1: Pathological features of polyomavirus-associated nephropathy.

Immunofluorescence: The results of immunofluorescence of $\operatorname{IgG}$, IgM, IgA, C3, C4, C1q, and C4d were negative.
Immunohistochemistry: A large aggregation of CD3, CD4, CD8, CD68 positive cells, CD3 $809 \pm 201 / \mathrm{mm}^{2}$, CD4 $348 \pm 110 / \mathrm{mm}^{2}$, CD8 $295 \pm 89 / \mathrm{mm}^{2}$, and CD68 $679.5 \pm 209 / \mathrm{mm}^{2}$, can be seen in renal interstitial from all renal pathology. In one patient combined with rejection, HLA-DR and high IL-2R expression were $19.6 \%$ and $148 / \mathrm{mm}^{2}$, respectively; in patients combined without rejection, HLADR and IL-2R expression were mostly less than 5\%. 31 patients were implemented with SV-40 large $\mathrm{T}$ antigen staining of renal tissues, positive scattered polyomavirus tubules can be seen in renal cortex and medulla, that is the nuclear of tubular epithelial cells was brown, the middle was transparent, and the peripheral was burr-like, and exfoliated cell polyomavirus expression can be seen in lumen of renal tubules (Figure 2A, 2B).

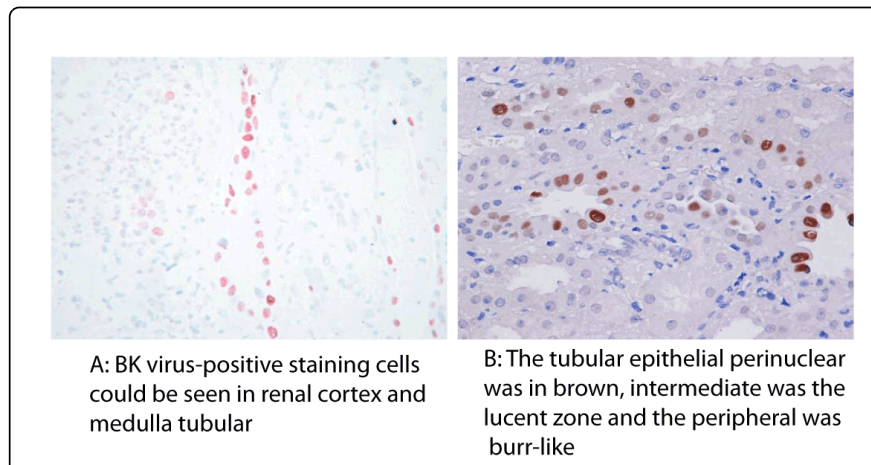

Figure 2: BK virus test in frozen section of renal tissues $(\mathrm{IH}, \times 400)$.

Ultrastructural characteristics of polyomavirus inclusion body: 31 cases of patients with PVAN were confirmed by electron microscopy under electron microscopy, virus-like particles arranged in dense crystalline with a diameter of $40 \sim 45 \mathrm{~nm}$ can be seen in renal tubular epithelial cells or endothelial cells (Figure 3A, 3B).

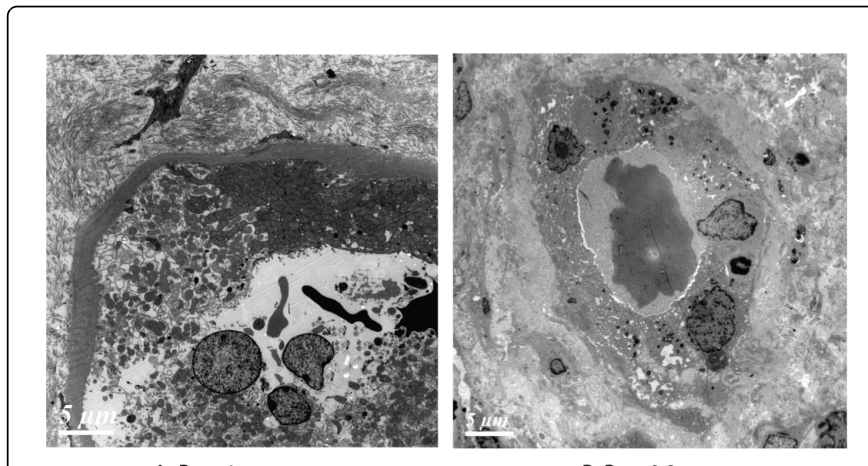

$\mathrm{A}: \mathrm{Bar}=1 \mu \mathrm{m}$

$B: B a r=0.2 \mu \mathrm{m}$

Figure 3: Ultra structural changes of polyomavirus-associated nephropathy.

Concentration of immunosuppressant and PVAN: 31 patients were carried out with three-point MPA AUC 0-12 examination when biopsy, and the results showed significantly higher than patients who 
were not infected with polyomavirus, $75.85 \pm 20.49(51.04-96.33) \mathrm{mg}$ h/L vs. $36.3 \pm 11.9$ (34.5-55.1) $\mathrm{mg}$. $\mathrm{h} / \mathrm{L}$, respectively, $\mathrm{P}<0.001$. FK506 trough concentrations were also significantly higher than that in control group, $9.28 \pm 2.38(9.8 \sim 11.05)$ ug/L vs. $5.95 \pm 1.14(5.6-7.2) \mathrm{ug} /$ $\mathrm{L}, \mathrm{P}<0.05$.

PVAN follow-up treatment: After 31 patients were diagnosed as PVAN, FK506 was reduced, triptolide and MMF were stopped and converted to leflunomide $(40 \mathrm{mg} / \mathrm{d})$ to maintain the therapy. The longest follow-up time was 8 years, with an average of $6.8 \pm 1.2$ years. Renal allograft function was stable and serum creatinine was remained at $1.46-4.57 \mathrm{mg} / \mathrm{dl}$, but the serum creatinine levels of most patients could not be reduced to the level before the onset. There were 3 cases of allograft loss, 16 cases of improved, 10 cases of stable, and 2 cases of deteriorated, and the average serum creatinine doubling time was longer than $22.4 \pm 11.6$ months. There was no death during the whole follow-up period.

\section{Discussion}

According to the data provided, 351 patients had polyomavirus large $\mathrm{T}$ antigen staining, 31 of which were positively diagnosed as having polyomavirus associated nephropathy thus leading to a positive rate of $8.8 \%$ which was consistent with that reported in international data [16]. The result of the analysis shows that PVAN mostly occurs between 3 and 13 months after renal transplantation with the mean value of 10 months. The disease does not have any typical clinical symptoms and is usually characterized by asymptomatic elevated serum creatinine with urinalysis parameters indicative of tubular injury, such as elevating NAG enzyme and urine RBP protein level. Positive Decoy cells can be found in the urinalysis with a positive rate of 12.9. It is shown that in addition to changes in amount and morphous of exfoliative cell in urine, other reasons such as adenovirus infection could also result in positive decoy cells in urine. Therefore, the urinalysis is of low specificity, and can be used as a screening indicator for PVAN patients [17-20]. As to why PVAN patients are mainly characterized by tubular injury but not proteinuria and erythrocyturia still requires further studies. And the most probably reason is that polyomavirus may be latent in renal tubular epithelial cell sand urinary tract transitional epithelial cells after in apparent infection and massive virus replication may occur when the host experiences decreased immunity which finally leads to tubular injury $[2,9]$.

Confirmed diagnosis of PVAN should be made on the basis of transplant renal biopsy $[8,21]$. The typical pathological manifestation of PVAN is presentation of inclusion bodies of basophilic polyomavirus with different phenotypes in renal tubular epithelial cell nucleus. The infected renal tubular epithelial cells drop out toward renal tubular lumen leading to bare plaque on basement membrane (Figure 1a, 1b).The prominent pathological manifestation of the renal biopsy is multifocal aggregation of massive infiltrating cells in renal interstitium which are mainly mononuclear cells and plasma cells. Tubulitis, multifocal tubule basement membrane thickening and atrophy can be observed as the most common cases with surrounding tubules (Figure 1c, 1d).Some pathological changes are mainly fibrosis which is only seen in a small number of cells infected with polyomavirus. 31 PVAN patients were confirmed by electron microscopy. The electron microscopy revealed dense crystalline-like arranged viral particle with diameters of $40-45 \mathrm{~nm}$ in renal tubular epithelial cells and renal tubular endothelial cells. Hirsch et al. [7] classified the pathological process of PVAN into 3 stages through semi- quantitative assessment based on morphological changes in cells, inflammatory cell infiltration, tubular atrophy and interstitial fibrosis: Stage I: characterized by positive large $\mathrm{T}$ antigen staining in focal tubular epithelial cells and, minimal cytopathological changes in viral inclusion bodies, without extensive necrosis and inflammatory infiltration. Stage II: characterized by extensive multifocal and diffused cytopathological changes, necrosis complicated with inflammatory reactions, and initial signs of interstitial fibrosis. Infiltrating inflammatory cells include polymorphocytes, mononuclear cells and plasma cells. Stage III: characterized by renal interstitial fibrosis, cicatricose even calcification and flatten and atrophy of tubular cells. Stage II is the most common type of PVAN observed in the analysis. Therefore, it is of extremely important clinical value of periodic transplant renal biopsy in patients with renal allograft dysfunction in early discovery and staging of PVAN.

We interpret tubulitis in the context of various light microscopical and immunohistochemical findings before rendering two concomitant diagnoses: acute rejection and PVAN. In our opinion, interstitial inflammation in the setting of PVAN may have different causes and the diagnosis of acute rejection can be difficult. Arteries and glomerular capillary tufts typically do not harbor polyomaviruses. Thus, transplant endarteritis, fibrinoid arterial necrosis or glomerulitis associated with tubulitis mark acute rejection. Significant renal abnormalities were noted at renal biopsy in 31 patients of which 1 patient was initially diagnosed as acute rejection. Since the early pathological features of PVAN are usually similar with that of interstitial nephritis, it is difficult to distinguish PVAN and acute rejection solely by routine staining and light microscopy especially mild rejection. Therefore, it is very necessary to use a combination of light microscopy and immunohistochemical staining. SV-40 large T antigen staining of renal tissues which has a great specificity is used in this study. Immunohistochemical staining of renal allograft tissues with SV-40 large T antigen is the well-recognized "golden standard" for diagnosis of PVAN [22]. In this study, SV-40 large T antigen staining of renal tissues of all the patients shows that there are scattered positive tubular polyomaviruses in the renal cortex and medulla, i.e. brown color around renal tubular epithelial cellnucleus, lucent area in the middle and burr-like periphery. In addition, polyomavirus expression in drop out cells can also be seen in renal tubular lumen (Figure 2). Furthermore, acute rejection concomitant with PVAN should be distinguished with non-specific interstitial change secondary to virus, as severe viral infection under high burden could lead to tubular epithelial cell necrosis, polymorphonuclear leukocytes and plasma cells infiltration. This is possibly one of the reasons for high misdiagnosis rate. It is interesting to find that all renal allograft immunofluorescence of IgG, IgM, IgA, C3, C4, C1q, and C4d staining were negative, and a large aggregation of CD3, CD4, CD8, CD68 positive cells, CD3 $809 \pm$ $201 / \mathrm{mm}^{2}$, CD4 $348 \pm 110 / \mathrm{mm}^{2}$, CD8 $295 \pm 89 / \mathrm{mm}^{2}$, and CD68 679.5 $\pm 209 / \mathrm{mm}^{2}$, can be seen in renal interstitial from all renal pathology. HLA-DR and high IL-2R expression in the patient combined with rejection were $19.6 \%$ and $148 / \mathrm{mm}^{2}$, respectively. HLA-DR and IL-2R expression in the patients combined without rejection were mostly less than 5\%. Therefore, HLA-DR, IL-2R expression in the allograft and C4d staining were important indicators for different PVAN and acute rejection, and may serve as additional markers to confirm the diagnosis of rejection [23].

It is reported that long-term high-dose application of FK506, MMF and other immunosuppressive agents can promote polyomavirus replication [7,24-26]. Several study sites for renal transplantation find that incidence of PVAN is significantly increased when previous 
immunosuppressants regimen of cyclosporine A plus azathioprine (Aza) is switched to tacrolimus and mycophenolate mofetil. Treatment with high dose of tacrolimus (whole blood concentration at $8 \mu \mathrm{g} / \mathrm{L}$ ) or mycophenolate mofetil makes the incidence of PVAN 13 times higher. Results from a prospective study suggest that the incidence of PVAN in renal transplant patients receiving tacrolimus and mycophenolate mofetil based immunosuppressive therapy is $5 \%$. Therefore, tacrolimus and mycophenolate mofetil based therapy may put patients in increased risk of PVAN. In all of the cases in this study, all the patients were given tacrolimus and mycophenolate mofetil except 1 patient who received cyclosporine A plus azathioprine plus Pred. Blood concentration of tacrolimus and MPA were both higher than that of control group. It requires further clinical observation with a large sample size to decide whether tacrolimus and MPA are high risk factors for PVAN. However, polyomavirus infection is not related to a certain immunosuppressant, but is more likely to have a relationship with the excessive immunosuppression in general.

Although it is reported [27-29] that cidofovir and leflunomide can be effectively used for antiviral treatment of PVAN, it is still a lack of accepted, standard treatment. For the treatment of PVAN, dose reduction of immunosuppressants was the core principle [9], All the patients diagnosed with PVAN reduced their tacrolimus dose and discontinued mycophenolate mofetil, i.e. they received low-dose tacrolimus in combination with leflunomide instead. The follow-up time is 5 years in maximum and half a year in minimum with the mean value of $26.60 \pm 13.76$ months. Data from follow-up indicates that most patients still have their creatinine level beyond that prior to the onset. There is 1 case of renal allograft dysfunction, 3 cases of improvement, 2 cases of stable disease and 3 cases of deterioration. The mean time to increased creatinine level is $>22.4 \pm 15.6$ months. No death has been reported at this time. Comparative studies with large sample size are needed to further investigate the long-term efficacy of leflunomide in the treatment PVAN [30].

In conclusions, high-dose immunosuppression or immune dysfunction is the main reason for the occurrence PVAN. Patients with PVAN are usually characterized by slowly progressive increase in serum creatinine with elevated renal tubular injury test parameters. Diagnosis of PVAN mainly depends on histopathology of renal allograft biopsy. Use of immunohistochemical staining with SV-40 large $\mathrm{T}$ antigen and electron microscopy will increase the confirmed diagnosis rate of PVAN. There is an extremely important clinical value of human leukocyte antigen DR, interleukin-2 receptor and C4d detection of allograft tissues in the diagnosis of PVAN. Most cases of disease progression can be controlled by leflunomide, but the longterm efficacy of the drug still requires further investigation.

\section{Acknowledgement}

The authors acknowledge Hui-Ping Chen and Cai-Hong Zeng for work in diagnostic pathology and $\mathrm{Dr}$ Zhaohong Chen for their technical supports.

\section{References}

1. Shuming J, Zheng T (2006) Polyomavirus-associated nephropathy after renal allograft. Chinese Journal of Nephrology Dialysis \& Transplantation 15: 572-578.

2. Costa C, Cavallo R (2012) Polyomavirus-associated nephropathy. World J Transplant 2: 84-94.
3. Jacobi J, Prignitz A, Büttner M, Korn K, Weidemann A, et al. (2013) BK viremia and polyomavirus nephropathy in 352 kidney transplants; risk factors and potential role of mTOR inhibition. BMC Nephrol 14: 207.

4. Hirsch HH, Randhawa P, AST Infectious Diseases Community of Practice (2013) BK polyomavirus in solid organ transplantation. Am J Transplant 13 Suppl 4: 179-188.

5. Renner FC, Dietrich H, Bulut N, Celik D, Freitag E, et al. (2013) The risk of polyomavirus-associated graft nephropathy is increased by a combined suppression of CD8 and CD4 cell-dependent immune effects. Transplant Proc 45: 1608-1610.

6. Lin MC, Wang M, Fang CY, Chen PL, Shen CH, et al. (2014) Inhibition of BK virus replication in human kidney cells by BK virus large tumor antigen-specific shRNA delivered by JC virus-like particles. Antiviral Res 103: $25-31$.

7. Hirsch HH, Vincenti F, Friman S, Tuncer M, Citterio F, et al. (2013) Polyomavirus BK replication in de novo kidney transplant patients receiving tacrolimus or cyclosporine: a prospective, randomized, multicenter study. Am J Transplant 13: 136-145.

8. John R, Herzenberg AM (2010) Our approach to a renal transplant biopsy. J Clin Pathol 63: 26-37.

9. Menter T, Mayr M, Schaub S, Mihatsch MJ, Hirsch HH, et al. (2013) Pathology of resolving polyomavirus-associated nephropathy. Am J Transplant 13: 1474-1483.

10. Ahuja M, Cohen EP, Dayer AM, Kampalath B, Chang CC, et al. (2001) Polyoma virus infection after renal transplantation. Use of immunostaining as a guide to diagnosis. Transplantation 71: 896-899.

11. Celik B, Shapiro R, Vats A, Randhawa PS (2003) Polyomavirus allograft nephropathy: sequential assessment of histologic viral load, tubulitis, and graft function following changes in immunosuppression. Am J Transplant 3: 1378-1382.

12. Racusen LC, Solez K, Colvin RB, Bonsib SM, Castro MC, et al. (1999) The Banff 97 working classification of renal allograft pathology. Kidney Int 55: 713-723.

13. Böhmig GA, Exner M, Habicht A, Schillinger M, Lang U, et al. (2002) Capillary C4d deposition in kidney allografts: a specific marker of alloantibody-dependent graft injury. J Am Soc Nephrol 13: 1091-1099.

14. Hassan S, Mittal C, Amer S, Khalid F, Patel A, et al. (2014) Currently recommended BK virus (BKV) plasma viral load cutoff of $\geq 4 \log 10 / \mathrm{mL}$ underestimates the diagnosis of BKV-associated nephropathy: a single transplant center experience. Transpl Infect Dis 16: 55-60.

15. Chen Huiping, Liu Zhihong, Zeng Lishi (2007) Polyomavirus-associated nephropathy. Chinese Journal of Nephrology Dialysis \& Transplantation 16: 389-393.

16. Namba Y, Moriyama T, Kyo M, Imamura R, Shi Y, et al. (2005) Prevalence, characteristics, and outcome of BK virus nephropathy in Japanese renal transplant patients: analysis in protocol and episode biopsies. Clin Transplant 19: 97-101.

17. Huang G, Chen WF, Wang CX, Fei JG, Deng SX, et al. (2013) Noninvasive tool for the diagnosis of polyomavirus BK-associated nephropathy in renal transplant recipients. Diagn Microbiol Infect Dis 75: 292-297.

18. Kroth LV, Henkin CS, Peres LD, Paganella MC, Mazzali M, et al. (2012) Prevalence of urinary decoy cells and associated risk factors in a Brazilian kidney, pancreas, and kidney-pancreas transplant population. Transplant Proc 44: 2394-2396.

19. Knight RJ, Gaber LW, Patel SJ, DeVos JM, Moore LW, et al. (2013) Screening for BK viremia reduces but does not eliminate the risk of BK nephropathy: a single-center retrospective analysis. Transplantation 95: 949-954.

20. Dadhania D, Snopkowski C, Ding R, Muthukumar T, Lee J, et al. (2010) Validation of noninvasive diagnosis of BK virus nephropathy and identification of prognostic biomarkers. Transplantation 90: 189-197.

21. Drachenberg CB, Papadimitriou JC, Hirsch HH, Wali R, Crowder C, et al. (2004) Histological patterns of polyomavirus nephropathy: correlation with graft outcome and viral load. Am J Transplant 4: 2082-2092. 
Citation: Shu-Ming JI, Ke-nan X, Jin-song C, Ji-qiu W, Dong-rui C, et al. (2014) Clinical and Pathological Study of Polyomavirus-Associated Nephropathy after Renal Transplantation. J Transplant Technol Res 4: 132. doi:10.4172/2161-0991.1000132

Page 6 of 6

22. Nickeleit V, Hirsch HH, Zeiler M, Gudat F, Prince O, et al. (2000) BKvirus nephropathy in renal transplants-tubular necrosis, MHC-class II expression and rejection in a puzzling game. Nephrol Dial Transplant 15: 324-332.

23. Ji Shuming, Li Leishi, Chen Huiping (1996) Re-evaluation of soluble interleukin-2 receptor used in the diagnosis of acute rejection. Chinese Journal of Nephrology Dialysis \& Transplantation 5: 17-20.

24. Brennan DC, Agha I, Bohl DL, Schnitzler MA, Hardinger KL, et al. (2005) Incidence of BK with tacrolimus versus cyclosporine and impact of preemptive immunosuppression reduction. Am J Transplant 5: 582-594.

25. Weiss AS, Gralla J, Chan L, Klem P, Wiseman AC (2008) Aggressive immunosuppression minimization reduces graft loss following diagnosis of BK virus-associated nephropathy: a comparison of two reduction strategies. Clin J Am Soc Nephrol 3: 1812-1819.

26. Ji SM, Wu D, Wen JQ, Xie KN, Cheng DR, et al. (2012) BK virus associated nephropathy after kidney transplantation. Zhongguo Zuzhi Gongcheng Yanjiu 16: 7411-7416.
27. Comoli P, Cioni M, Basso S, Gagliardone C, Potenza L, et al. (2013) Immunity to Polyomavirus BK Infection: Immune Monitoring to Regulate the Balance between Risk of BKV Nephropathy and Induction of Alloimmunity. Clin Dev Immunol 2013: 256923.

28. Hüttemann M, Shipkova M, Klett C, Hasche G, Wilhelm J, et al. (2013) Total and free plasma concentrations of the active metabolite of leflunomide in relation to therapeutic outcome in kidney transplant recipients with BK-virus nephropathy. Transplant Proc 45: 1611-1613.

29. Jung YH, Moon KC, Ha JW, Kim SJ, Ha IS, et al. (2013) Leflunomide therapy for BK virus allograft nephropathy after pediatric kidney transplantation. Pediatr Transplant 17: E50-54.

30. Wojciechowski D, Chanda R, Chandran S, Lee B, Webber A, et al. (2012) Ciprofloxacin prophylaxis in kidney transplant recipients reduces $\mathrm{BK}$ virus infection at 3 months but not at 1 year. Transplantation 94: 1117-1123. 